\title{
Dr Charles West (1816-98) of London and the cold syndrome
}

\author{
P M Dunn
}

Charles West was born in London, the son of a Baptist lay preacher. He was educated at a school run by his father and then apprenticed to a general practitioner in Amersham. In 1833 he entered St Bartholomew's Hospital and after studies there and in Bonn and Paris, took his MD in Berlin in 1837. After a short period of general practice in London he visited Dublin where he made a special study of midwifery. Returning to London he was appointed physician to the Royal Universal Dispensary for Women and Children, Lambeth, in 1842 and lecturer in midwifery at the Middlesex Hospital in 1845, and later also at St Bartholomew's Hospital (1848-61). His Lectures on Diseases of Infancy and Childhood, published in 1848, were a great success, passing through seven editions in Great Britain, five in America, and translations into nine languages. His Lectures on Diseases of Women, published in 1856, met with a similar success.

In 1850 there were still no special hospitals for children in this country, although there were 17 on the continent. In London, where nearly $50 \%$ of all deaths involved young children, only $1 \%$ of all hospital inpatients derived from this age group. In that year Dr West, supported by Dr Bence Jones, set about founding the Hospital for Sick Children in Great Ormond Street in a house formerly occupied by $\mathrm{Dr}$ Richard Mead. It opened on St Valentine's Day, 1852, with 10 beds, and for the next 23 years Charles West was its senior physician. He also played an active part in planning the new 120 bed hospital, opened to celebrate Queen Victoria's Jubilee in 1877.

The following extract on induration of the cellular tissue is taken from the 5th edition of the Lectures on the Diseases of Infancy and Childhood. ${ }^{1}$ In the preface to this work West states that it embodies the results of 1,200 recorded cases, and nearly 400 post-mortem examinations, collected from between 30,000 and 40,000 children who, during the past twenty-six years, have come under my care, either in public or in private practice'. His clinicopathological description of conditions we would now term sclerema neonatorum and the cold syndrome is remarkably 'modern'. Indeed the cold syndrome, described with its characteristic livid redness, oedema, and associated pulmonary haemorrhage, was really only rediscovered a hundred years later.

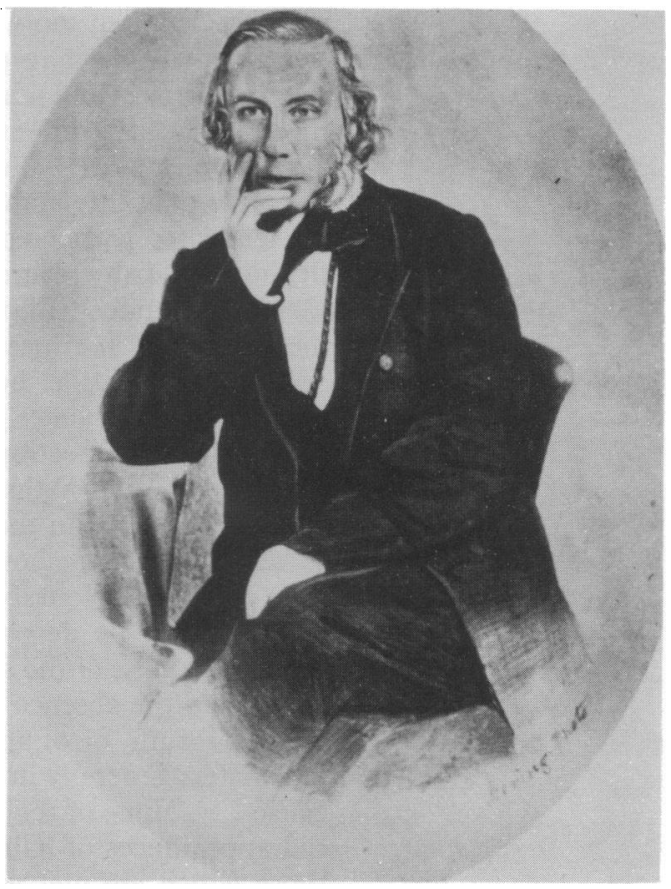

Dr Charles West, 1816-1898.

'Though very rare in this country, induration of the cellular tissue is extremely common in the foundling hospitals of the Continent, where so many causes contribute to depress the newborn infant's feeble powers. The children in whom it occurs are usually weakly, not seldom premature, and its first symptoms generally appear between the first and fifth day after birth, though occasionally they do not come on till later. In many instances a livid redness of the whole surface is obvious from birth; but the appearance of a circumscribed hard spot on one or other extremity, or on some prominent part of the face, as the end of the chin, or the cheek bone, is the first sign of the commencement of this affection. Other spots of a similar kind are soon discovered on different parts of the surface; and the body generally, and the hardened spots in particular, are found to present a temperature much below the natural warmth of the body. It appears, indeed, from M Roger's researches, that a general reduction of the temperature precedes the induration, or, at least, exists in a very marked degree, while the induration is still extremely slight. Sometimes, 
too, the premonitory loss of temperature may be perceived in weakly children without being succeeded by the appearance of spots of induration. This, however, is exceptional, and in the majority of instances the sinking of the temperature and the extension of the induration advance together, and the warmth of the surface may eventually fall from $100^{\circ}$ to $90^{\circ}, 80^{\circ}$, or even lower. If the induration become very extensive, it affects the integuments of the chest and the abdomen, as well as the extremities, and the body feels cold and stiff, as though it were frozen.

This condition is, as might be expected, attended with great impairment of the general health, and with a very remarkable degree of emaciation. Children suffering from it are extremely weak, often too weak to suck: their pulse is very small, their respiration abdominal, and their cry is faint and whimpering, wholly unlike that of a healthy infant. In some of the worst cases, too, a bloody fluid is discharged in considerable quantity from the nose and mouth. If the indurated parts be punctured, a small quantity of reddish serum escapes from them, though generally without much diminution of their previous hardness.

If the induration be at all general, death almost invariably takes place; and so great is the fatality of the affection, that, including even slight cases, five-sixths of those children who are attacked by it in the hospitals of Paris, die. In very slight cases, however, if the infant be at once placed in favourable circumstances, recovery need not be despaired of.

The hardness of the surface still persists after death, and the absence of any peculiarity in the effused serum, or of any sign of active disease, left writers generally in much perplexity as to its cause ... . None of the viscera present any morbid appearances of half so much importance as those which are met with in the lungs, a very great part of which displays those changes to which your attention has already been directed as characteristic of their deficient expansion. This condition of the lungs had been noticed and most carefully described many years ago, as one of the most striking attendants on induration of the cellular tissue. It was thought by some of those who described it to be the result of pneumonia . . . We, however, are aware that those appearances once thought to be the result of pneumonia, are in reality due to the unex- panded condition of the lung; and we can understand how it may happen, if children be exposed to cold immediately after birth, and then transferred to the ill-ventilated wards of a foundling hospital, and there fed with food far other than that which nature destined for them, that respiration may be but very imperfectly established; that their temperature may consequently fall, and the blood flowing in part through the unclosed foetal passages may stagnate in its course, may give rise to passive effusions into the great cavities of the body, and to an anasarcous swelling of the surface. There are, it is true, some peculiarities in this form of oedema, but not such as to invalidate the above explanation of the cause to which it is due.

The treatment of this affection implies the removal of every cause likely to induce it. Hence warmth stands foremost both as a curative and as a preventive measure. The warm bath may be resorted to as a means of raising the child's surface to a proper temperature, provided its extreme weakness do not contraindicate that measure. Gentle friction with warm oil is a means which has been tried for this purpose with advantage. The child should be nourished with breast-milk, even if it be too feeble to suck, and stimulants, of which whitewine whey is a very good one, will in many instances be needed. Defective respiration being the ultimate source of all the symptoms, the main principles of all your treatment must be the same as have already been laid down for your guidance in cases of atelektasis of the lung; and these it can hardly be necessary to recapitulate'.

Dr West became Senior Censor to the Royal College of Physicians in 1870 and delivered the Croonian lectures in 1854, the Lumleian lectures in 1871, and the Harveian oration in $\mathbf{1 8 7 4}$. Although a fine public speaker and an able man, he was not an easy colleague and he also fiercely opposed the admission of women to the medical profession. He married twice, first Miss Cartwright of Stroud by whom he had a son and a daughter, and second, a Miss Flon. He died in Paris in 1898 at the age of 82 .

1 West. Lectures on the diseases of infancy and childhood. 5th Ed. London: Longman, Green, Longman, Roberts and Green, 1865 . 